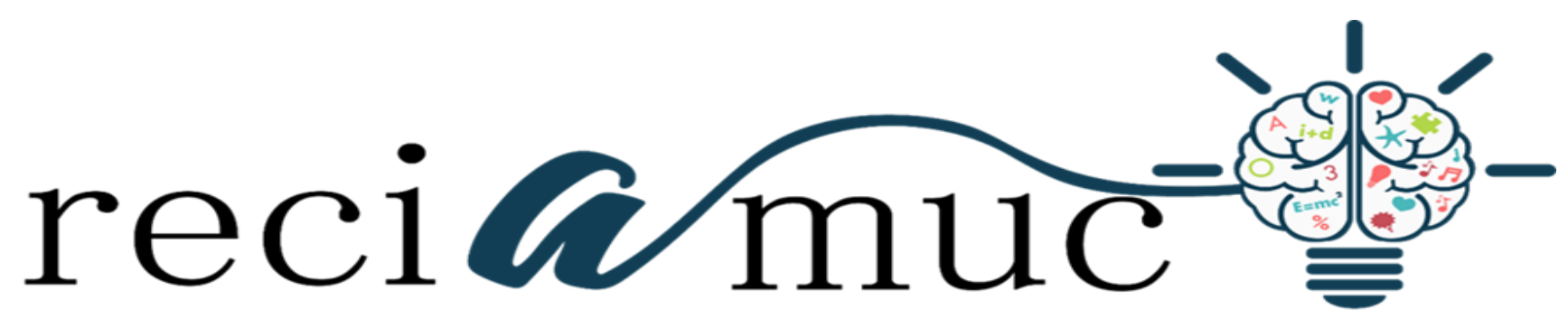

Revista cientifica de investigación actualización del mundo de las ciencias

Jairo Fernando Bailón Jiménez ${ }^{\text {a. }}$ Rebeca Isabel Lozano Vera ${ }^{\text {b; }}$ Piedad Shirley

Barragán Villafuerte ${ }^{\mathrm{c}}$; Karen Tatiana Mora Lino ${ }^{\mathrm{d}}$

Tratamiento y complicaciones de colecistitis calculosa aguda

Treatment and complications of acute calculus colecistitis

Revista Científica de Investigación actualización del mundo de las Ciencias. Vol. 3

núm., 2, abril, ISSN: 2588-0748, 2018, pp. 296-317

DOI: $10.26820 / \mathrm{reciamuc/3.(2).abril.2019.296-317}$

URL: http://reciamuc.com/index.php/RECIAMUC/article/view/341

Código UNESCO: 3205 Medicina Interna

Tipo de Investigación: Artículo de Revisión

(C) RECIAMUC; Editorial Saberes del Conocimiento, 2019

Recibido: 15/01/2019

Aceptado: 07/02/2019

Publicado: 01/04/2019

Correspondencia: jbailonj.91@gmail.com

a. Médico; Investigador Independiente; Guayaquil, Ecuador; jbailonj.91@ gmail.com

b. Médico; Investigador Independiente; Guayaquil, Ecuador; rilozanoczs5@gmail.com

c. Médico; Investigador Independiente; Guayaquil, Ecuador; barraganvillafuerte@ gmail.com

d. Médico; Investigador Independiente; Guayaquil, Ecuador; taty_2101@ hotmail.com 


\section{Tratamiento y complicaciones de colecistitis calculosa aguda}

Vol. 3, núm. 2., (2019)

Jairo Fernando Bailón Jiménez; Rebeca Isabel Lozano Vera; Piedad Shirley Barragán

Villafuerte; Karen Tatiana Mora Lino

\section{RESUMEN}

La colecistitis calculosa aguda es una afección que generalmente ocurre debido a un estado clínico grave, es relativamente rara y difícil de diagnosticar. Una patología que presenta etiología multifactorial. Este artículo hace mención a la colecistitis calculosa aguda, su diagnóstico y las complicaciones que esta enfermedad desencadena, así como también, los tratamientos aplicados a los pacientes. La investigación se realiza por medio de una revisión bibliográfica que se enfoca en temas relacionados al de interés y que brindaron aportes significativos. La tasa de mortalidad de pacientes con enfermedad aguda de colecistitis calculosa sigue siendo alta y se debe principalmente a las condiciones médicas subyacentes graves y la rápida progresión de la enfermedad a gangrena y perforación. La colecistitis aguda es una patología en la que la pared de la vesícula biliar se inflama siendo los cálculos biliares el factor desencadenante primario en casi la mayoría de las causas de colecistitis, están presentes en más del 10\% de la población y su presencia aumenta con la edad.

Palabras claves: Colecistitis; Colecistitis Aguda; Vesícula Biliar; Tratamiento; Complicaciones. 


\title{
Tratamiento y complicaciones de colecistitis calculosa aguda
}

Vol. 3, núm. 2., (2019)

Jairo Fernando Bailón Jiménez; Rebeca Isabel Lozano Vera; Piedad Shirley Barragán Villafuerte; Karen Tatiana Mora Lino

\begin{abstract}
Acute calculous cholecystitis is a condition that usually occurs due to a serious clinical condition, it is relatively rare and difficult to diagnose. A pathology that presents multifactorial etiology. This article mentions acute calculous cholecystitis, its diagnosis and the complications that this disease triggers, as well as the treatments applied to patients. The research is carried out through a bibliographic review that focuses on topics related to the one of interest and that provided significant contributions. The mortality rate of patients with acute calculous cholecystitis disease remains high and is mainly due to serious underlying medical conditions and the rapid progression of the disease to gangrene and perforation. Acute cholecystitis is a pathology in which the wall of the gallbladder becomes inflamed with gallstones being the primary trigger in almost all causes of cholecystitis, they are present in more than $10 \%$ of the population and their presence increases with age.
\end{abstract}

Key words: Cholecystitis; Acute Cholecystitis; Gallbladder; Treatment; Complications. 


\section{Tratamiento y complicaciones de colecistitis calculosa aguda}

Vol. 3, núm. 2., (2019)

Jairo Fernando Bailón Jiménez; Rebeca Isabel Lozano Vera; Piedad Shirley Barragán

Villafuerte; Karen Tatiana Mora Lino

\section{Introducción.}

La colecistitis calculosa aguda es una complicación de la colelitiasis, una afección que afecta a más de 20 millones de estadounidenses anualmente y resulta en costos directos de más de \$ 6.3 mil millones. La mayoría de los pacientes con cálculos biliares son asintomáticos. De tales pacientes, el cólico biliar se desarrolla en 1 a $4 \%$ anualmente, y la colecistitis aguda se desarrolla uniformemente en aproximadamente el $20 \%$ de estos pacientes sintomáticos si no se tratan. (Carter, Cox, \& Polk, 2013)

Los pacientes que presentan esta patología tienden a ser algo mayores que aquellos con colelitiasis sintomática sin complicaciones. La mayoría de los pacientes con colecistitis aguda han tenido ataques de cólico biliar, pero algunos no han tenido síntomas biliares previos. Después de un ataque inicial de colecistitis aguda, los ataques adicionales de dolor o inflamación son comunes. En una pequeña proporción de pacientes, la colecistitis aguda puede coexistir con coledocolitiasis, colangitis o pancreatitis de cálculos biliares. (Gurusamy \& Samraj, 2006)

Alrededor de 120.000 colecistectomías se realizan anualmente para la colecistitis aguda en los Estados Unidos. Sin embargo, la incidencia de colecistitis aguda parece estar disminuyendo debido a la mayor aceptación por parte de los pacientes de la colecistectomía laparoscópica como tratamiento para los cálculos biliares sintomáticos. Aproximadamente el $60 \%$ de los pacientes con colecistitis aguda son mujeres. Sin embargo, la colecistitis aguda se desarrolla en los hombres con más frecuencia de lo que se esperaría de la prevalencia relativa de cálculos biliares (aproximadamente la mitad que en las mujeres), siendo más grave en los hombres. (Yeatman, 2009) 


\section{Tratamiento y complicaciones de colecistitis calculosa aguda}

Vol. 3, núm. 2., (2019)

Jairo Fernando Bailón Jiménez; Rebeca Isabel Lozano Vera; Piedad Shirley Barragán Villafuerte; Karen Tatiana Mora Lino

En pacientes con diabetes que tienen cálculos biliares sintomáticos, la colecistitis aguda parece desarrollarse con mayor frecuencia que en pacientes sin diabetes, y es más probable que tales pacientes tengan complicaciones de colecistitis aguda cuando ocurre.

Más del 90\% de los casos de colecistitis aguda están asociados con colelitiasis (colecistitis calculosa aguda). Los elementos clave en la patogénesis parecen ser una obstrucción del conducto cístico en presencia de bilis sobresaturada con colesterolterol. Una breve impactación puede causar dolor solamente, pero si la impactación se prolonga durante muchas horas, puede producirse inflamación. Con la inflamación, la vesícula se agranda, se tensa y se enrojece, y se puede engrosar la pared y desarrollar un exudado de líquido pericolecítico. La inflamación es inicialmente estéril en la mayoría de los casos, pero la infección secundaria con microorganismos de la familia Enterobacteriaceae o con enterococos o anerobios ocurre en la mayoría de los pacientes. (Claesson, Holmlund, \& Mätzsch, 2014). La pared de la vesícula biliar puede sufrir necrosis y gangrena (colecistitis gangrenosa). La superinfección bacteriana con organismos formadores de gases puede conducir a gases en la pared o la luz de la vesícula biliar (colecistitis enfisematosa). Sin el tratamiento adecuado, la vesícula biliar puede perforar, con el desarrollo de un absceso en el cuadrante superior derecho o el hígado o peritonitis generalizada.

\section{Métodos y materiales.}

Para el desarrollo de este proceso investigativo, se plantea como metodología la encaminada hacia una orientación científica particular que se encuentra determinada por la necesidad de indagar en forma precisa y coherente una situación, en tal sentido (Davila, 2015) 


\section{Tratamiento y complicaciones de colecistitis calculosa aguda}

Vol. 3, núm. 2., (2019)

Jairo Fernando Bailón Jiménez; Rebeca Isabel Lozano Vera; Piedad Shirley Barragán

Villafuerte; Karen Tatiana Mora Lino

define la metodología "como aquellos pasos previos que son seleccionados por el investigador para lograr resultados favorables que le ayuden a plantear nuevas ideas”. (p.66)

Lo citado por el autor, lleva a entender que el desarrollo de la acción investigativa busca simplemente coordinar acciones enmarcadas en una revisión bibliográfica con el fin de complementar ideas previas relacionadas Tratamiento y complicaciones de colecistitis calculosa aguda, a través de una revisión de literatura, para así finalmente elaborar un cuerpo de consideraciones generales que ayuden a ampliar el interés propuesto.

\section{Tipo de investigación.}

Dentro de toda práctica investigativa, se precisan acciones de carácter metodológico mediante las cuales, se logra conocer y proyectar los eventos posibles que la determinan, así como las características que hacen del acto científico un proceso interactivo ajustado a una realidad posible de ser interpretada. En este sentido, se puede decir, que la presente investigación corresponde al tipo documental, definido por Castro (2016), "se ocupa del estudio de problemas planteados a nivel teórico, la información requerida para abordarlos se encuentra básicamente en materiales impresos, audiovisuales y /o electrónicos”. (p.41).

En consideración a esta definición, la orientación metodológica permitió la oportunidad de cumplir con una serie de actividades inherentes a la revisión y lectura de diversos documentos donde se encontraron ideas explicitas relacionadas con los tópicos encargados de identificar a cada característica insertada en el estudio. Por lo tanto, se realizaron continuas interpretaciones con el claro propósito de revisar aquellas apreciaciones o investigaciones propuestas por diferentes 


\section{Tratamiento y complicaciones de colecistitis calculosa aguda}

Vol. 3, núm. 2., (2019)

Jairo Fernando Bailón Jiménez; Rebeca Isabel Lozano Vera; Piedad Shirley Barragán Villafuerte; Karen Tatiana Mora Lino

investigadores relacionadas con el tema de interés, para luego dar la respectiva argumentación a los planteamientos, en función a las necesidades encontradas en la indagación.

\section{Fuentes documentales.}

El análisis correspondiente a las características que predomina en el tema seleccionado, llevan a incluir diferentes fuentes documentales encargadas de darle el respectivo apoyo y en ese sentido cumplir con la valoración de los hechos a fin de generar nuevos criterios que sirven de referencia a otros procesos investigativos. Para (CASTRO, 2016) las fuentes documentales incorporadas en la investigación documental o bibliográfica, "representa la suma de materiales sistemáticos que son revisados en forma rigurosa y profunda para llegar a un análisis del fenómeno".(p.41). Por lo tanto, se procedió a cumplir con la realización de una lectura previa determinada para encontrar aquellos aspectos estrechamente vinculados con el tema, con el fin de explicar mediante un desarrollo las respectivas apreciaciones generales de importancia.

\section{Técnicas para la recolección de la información.}

La conducción de la investigación para ser realizada en función a las particularidades que determinan a los estudios documentales, tiene como fin el desarrollo de un conjunto de acciones encargadas de llevar a la selección de técnicas estrechamente vinculadas con las características del estudio. En tal sentido, (Bolívar, 2015), refiere, que es "una técnica particular para aportar ayuda a los procedimientos de selección de las ideas primarias y secundarias”. (p. 71). 


\section{Tratamiento y complicaciones de colecistitis calculosa aguda}

Vol. 3, núm. 2., (2019)

Jairo Fernando Bailón Jiménez; Rebeca Isabel Lozano Vera; Piedad Shirley Barragán

Villafuerte; Karen Tatiana Mora Lino

Por ello, se procedió a la utilización del subrayado, resúmenes, fichaje, como parte básica para la revisión y selección de los documentos que presentan el contenido teórico. Es decir, que mediante la aplicación de estas técnicas se pudo llegar a recoger informaciones en cuanto a la revisión bibliográfica de los diversos elementos encargados de orientar el proceso de investigación. Tal como lo expresa, (Bolívar, 2015) "las técnicas documentales proporcionan las herramientas esenciales y determinantes para responder a los objetivos formulados y llegar a resultados efectivos" (p. 58). Es decir, para responder con eficiencia a las necesidades investigativas, se introdujeron como técnica de recolección el método inductivo, que hizo posible llevar a cabo una valoración de los hechos de forma particular para llegar a la explicación desde una visión general.

Asimismo, se emplearon las técnicas de análisis de información para la realización de la investigación que fue ejecutada bajo la dinámica de aplicar diversos elementos encargados de determinar el camino a recorrer por el estudio, según, (Bolívar, 2015) las técnicas de procesamiento de datos en los estudios documentales "son las encargadas de ofrecer al investigador la visión o pasos que debe cumplir durante su ejercicio, cada una de ellas debe estar en correspondencia con el nivel a emplear" (p. 123). Esto indica, que para llevar a cabo el procesamiento de los datos obtenidos una vez aplicado las técnicas seleccionadas, tales como: fichas de resumen, textual, registros descriptivos entre otros, los mismos se deben ajustar al nivel que ha sido seleccionado.

\section{Resultados:}

El síntoma principal de la colelitiasis sin complicaciones es el cólico biliar, causado por la obstrucción del cuello de la vesícula biliar por un cálculo. El dolor es característico severo y se 


\section{Tratamiento y complicaciones de colecistitis calculosa aguda}

Vol. 3, núm. 2., (2019)

Jairo Fernando Bailón Jiménez; Rebeca Isabel Lozano Vera; Piedad Shirley Barragán Villafuerte; Karen Tatiana Mora Lino

localiza en el epigastrio o en el cuadrante superior derecho. Frecuentemente aparece luego de la ingesta de alimentos o aparece de noche. Los pacientes comúnmente tienen dolor que se irradia hacia la espalda, acompañado de náuseas y vómitos. La colecistitis aguda generalmente comienza con un ataque de cólico biliar, a menudo en un paciente que ha tenido ataques previos, pero el dolor persiste y se localiza en el cuadrante superior derecho. El nivel de bilirrubina puede aumentar a $4 \mathrm{mg}$ por decilitro (68 $\mu \mathrm{mol}$ por litro) en ausencia de complicaciones. Sin embargo, los autores (Roslyn, DenBesten, Thompson, \& Silverman, 2010) dicen que es poco común; y que cuando está presente, se debe levantar sospechas de coledocolitiasis concomitante, y tomar en consideración la presencia del síndrome de Mirizzi u obstrucción del conducto biliar como resultado de la compresión externa de un cálculo en la vesícula biliar o el conducto cístico u otra complicación, como la perforación de la vesícula biliar.

La inflamación en el cuadrante superior derecho son signos frecuentes. Una masa palpable está presente en una cuarta parte de los pacientes después de 24 horas de síntomas, pero rara vez se presenta temprano en el curso clínico. El signo de Murphy, la detención de la inspiración mientras se palpa la vesícula biliar durante una respiración profunda, puede ser útil, particularmente cuando no hay sensibilidad directa (por ejemplo, en un caso de sub-revestimiento). Ocasionalmente, la colecistitis aguda puede causar sepsis sistémica e insuficiencia orgánica, generalmente en el contexto de colecistitis gangrenosa o enfisematosa. La fiebre y una elevación en el recuento de glóbulos blancos se describen clásicamente en pacientes con colecistitis aguda, pero puede estar ausente uno o ambos. (Juvonen, Kiviniemi, Niemelä, \& Kairaluoma, 2007). 


\section{Tratamiento y complicaciones de colecistitis calculosa aguda}

Vol. 3, núm. 2., (2019)

Jairo Fernando Bailón Jiménez; Rebeca Isabel Lozano Vera; Piedad Shirley Barragán

Villafuerte; Karen Tatiana Mora Lino

Un nivel elevado de amilasa sérica sugiere pancreatitis biliar concomitante o colecistitis gangrenosa. En los pacientes de edad avanzada, los retrasos en el diagnóstico son comunes, ya que los únicos síntomas pueden ser un cambio en el estado mental o una disminución de la ingesta de alimentos, y el examen físico y los índices de laboratorio pueden ser normales. (Morrow, Thompson, \& Wilson, 2008)

Estudios de imagenología.

La ecografía abdominal y la gammagrafía hepatobiliar son los estudios de imagen más utilizados en el diagnóstico de colecistitis. La ecografía detecta la colelitiasis en aproximadamente el $98 \%$ de los pacientes como se observa en la figura $\mathrm{N}^{\circ} 1$. La colecistitis calculosa aguda se diagnostica radiológicamente por la presencia concomitante de engrosamiento de la pared de la vesícula biliar (5 $\mathrm{mm}$ o más), líquido pericolequístico o sensibilidad directa cuando la sonda se empuja contra la vesícula biliar (signo de Murphy ultrasonográfico). 


\section{Tratamiento y complicaciones de colecistitis calculosa aguda}

Vol. 3, núm. 2., (2019)

Jairo Fernando Bailón Jiménez; Rebeca Isabel Lozano Vera; Piedad Shirley Barragán Villafuerte; Karen Tatiana Mora Lino

\section{Figura 1. Imágenes ultrasonográficas de tres vesículas}

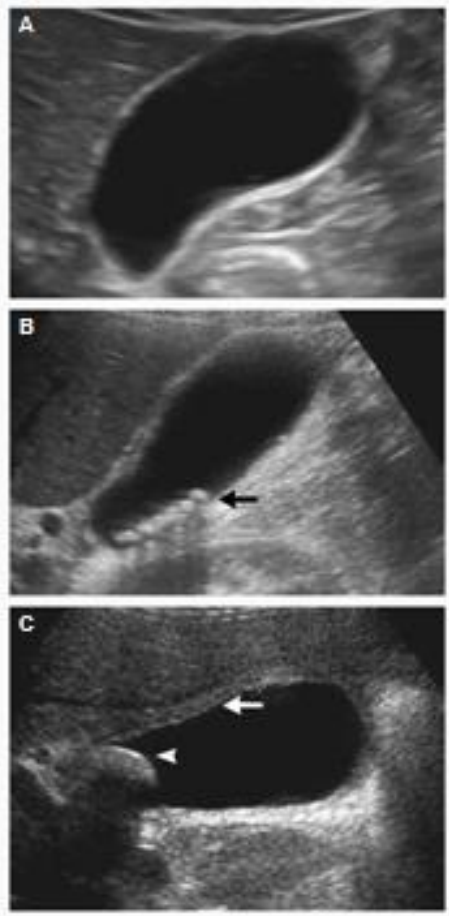

Fuente: (Ralls, 2013)

En la figura se observa una vesícula biliar sonolúcida normal (Panel A) se caracteriza por una pared delgada y la ausencia de sombras acústicas. En un paciente con cálculos biliares sintomáticos (Panel B), la vesícula contiene pequeños objetos ecogénicos con sombras acústicas posteriores que son típicas de los cálculos biliares (flecha), con un grosor de pared normal. En un paciente con colecistitis calculosa aguda (Panel C), el engrosamiento es visible en la pared de la vesícula biliar (flecha), junto con un gran cálculo biliar (punta de flecha).

En un estudio en el que participaron 497 pacientes con sospecha de colecistitis aguda, el valor predictivo positivo de la presencia de cálculos y un signo de Murphy ecográfico positivo fue del 92\%, y el de cálculos y engrosamiento de la pared de la vesícula biliar fue del 95\% .El 


\section{Tratamiento y complicaciones de colecistitis calculosa aguda}

Vol. 3, núm. 2., (2019)

Jairo Fernando Bailón Jiménez; Rebeca Isabel Lozano Vera; Piedad Shirley Barragán

Villafuerte; Karen Tatiana Mora Lino

predictivo valor negativo de la ausencia de cálculos combinados con una pared normal de la vesícula biliar o un signo negativo de Murphy fue del 95\%. (Ralls, 2013)

La gammagrafía hepatobiliar implica la inyección intravenosa de análogos de ácido iminodiacético marcados con tecnecio, que se excretan en la bilis. La ausencia de llenado de la vesícula biliar dentro de los 60 minutos posteriores a la administración del marcador indica la obstrucción del conducto cístico y tiene una sensibilidad del 80 al 90\% para la colecistitis aguda como se observa en la figura $\mathrm{N}^{\circ} 2$.

Figura 2. Gammagrafía hepatobiliar.
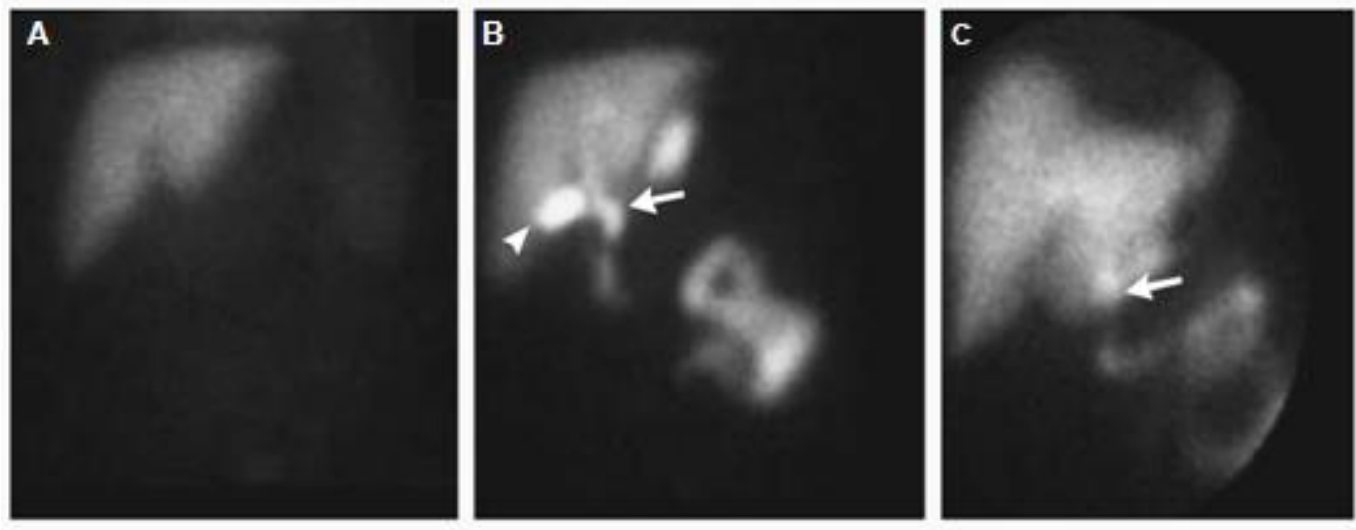

Fuente: (Ralls, 2013)

En la figura se observa, en el Panel A, un hígado normal es visible 10 minutos después de la inyección intravenosa de un análogo marcado con tecnecio de ácido iminodiacético. En el Panel B, a los 55 minutos después de la inyección del marcador, se puede ver el llenado del conducto biliar (flecha) y la vesícula biliar (punta de flecha). En el Panel C, 1 hora después de la inyección del marcador en un paciente con colecistitis aguda y obstrucción del conducto cístico, se llena el conducto biliar (flecha) pero no se llena la vesícula biliar. 


\section{Tratamiento y complicaciones de colecistitis calculosa aguda}

Vol. 3, núm. 2., (2019)

Jairo Fernando Bailón Jiménez; Rebeca Isabel Lozano Vera; Piedad Shirley Barragán Villafuerte; Karen Tatiana Mora Lino

La tasa de falsos positivos del 10 al 20\% se explica en gran medida por la obstrucción del conducto cístico inducida por la inflamación crónica, aunque en algunos casos las vesículas normales no se llenan como resultado de una resistencia insuficiente en el esfínter de Oddi. La especificidad de la prueba puede mejorarse mediante la administración intravenosa de morfina, que induce espasmos de este esfínter. (Juweid \& Mozley, 2003) Cuando el conducto cístico es patente (es decir, sin colecisistitis), la vesícula biliar normalmente se visualiza dentro de 30 minutos.

Cuando el llenado de la vesícula biliar se produce dentro de los 30 minutos, la tasa de falsos negativos (es decir, la presencia de colecistitis a pesar de los resultados negativos) es de solo $0.5 \%$, pero el llenado entre 30 minutos y 4 horas se asocia con tasas negativas falsas de 15 a $20 \%$. El "signo del borde" es un rubor de aumento de la radioactividad pericolequística, que está presente en aproximadamente el $30 \%$ de los pacientes con colecistitis aguda y en aproximadamente el $60 \%$ con colecistitis gangrenosa aguda. (Hicks, Kelly, \& V, 2011)

En las comparaciones de la ecografía y la gammagrafía hepatobiliar en pacientes con sospecha de colecistitis aguda, la gammagrafía tuvo una especificidad significativamente mayor y una mayor precisión que la ecografía. Sin embargo, la ecografía generalmente se ve favorecida como la primera prueba debido a la disponibilidad inmediata, la facilidad, la falta de interferencia de los niveles elevados de bilirrubina sérica (ya que la colestasis interfiere con la excreción biliar de los agentes utilizados en la gammagrafía), la ausencia de radiación ionizante y la capacidad de proporcionar información sobre la presencia de piedras. Cada vez más, los médicos de de emergencia están siendo entrenados en el uso de la ecografía. (Blaivas \& Adhikari, 2007). La 


\section{Tratamiento y complicaciones de colecistitis calculosa aguda}

Vol. 3, núm. 2., (2019)

Jairo Fernando Bailón Jiménez; Rebeca Isabel Lozano Vera; Piedad Shirley Barragán

Villafuerte; Karen Tatiana Mora Lino

gammagrafía hepatobiliar generalmente se reserva para el $20 \%$ de los pacientes en los que el diagnóstico no está claro después de la ecografía.

\section{Diagnóstico y grado.}

El estándar de oro para el diagnóstico es el examen patológico de la vesícula biliar. Existe controversia sobre los criterios óptimos para el diagnóstico clínico. La Tabla $\mathrm{N}^{\mathrm{o}} 1$ resume un conjunto recientemente recomendado de criterios de diagnóstico, llamados Directrices de Tokyo. (Takada, Kawarada, \& Nimura, 2007). Las limitaciones de estos criterios es que la condición de los pacientes con pocos síntomas sistémicos tiende a ser subdiagnosticados y que las pruebas de el nivel de proteína $\mathrm{C}$ reactiva no se usa comúnmente para el diagnóstico de cistitis aguda de cole. La conferencia de consenso de Tokio también clasificó la gravedad de la colecistitis aguda con el objetivo de guiar la terapia, en particular la colecistectomía (Tabla 2), aunque ningún estudio prospectivo ha determinado el desglose de los casos que caen en las tres categorías de la clasificación, la gran mayoría de los casos son leves. 


\section{Tratamiento y complicaciones de colecistitis calculosa aguda}

Vol. 3, núm. 2., (2019)

Jairo Fernando Bailón Jiménez; Rebeca Isabel Lozano Vera; Piedad Shirley Barragán Villafuerte; Karen Tatiana Mora Lino

Tabla 1. Criterios de diagnóstico para colecistitis aguda, según las guías de Tokio.

Manifestaciones clínicas.

Síntomas y signos locales.

Signo de Murphy.

Dolor o sensibilidad en el cuadrante superior derecho Masa en el cuadrante superior derecho.

Signos sistémicos.

Fiebre.

Leucocitosis.

Nivel elevado de proteína $\mathrm{C}$ reactiva.

Hallazgos de imagen.

Un hallazgo confirmatorio en la ecografía o gammagrafía hepatobiliar Diagnóstico.

La presencia de un signo o síntoma local, un signo sistémico y un hallazgo confirmatorio en una prueba de imagen.

Fuente: (Takada, Kawarada, \& Nimura, 2007)

Tabla $N^{a}$ 2. Clasificación de gravedad para la colecistitis aguda, según las directrices de Tokio.

\begin{tabular}{|l|l|}
\hline \multicolumn{1}{|c|}{ Grado } & \multicolumn{1}{c|}{ Criterio } \\
\hline Leve (grado 1) & $\begin{array}{l}\text { Colecistitis aguda que no cumple los criterios para } \\
\text { una inflamación leve de la vesícula biliar de grado } \\
\text { más grave, sin disfunción orgánica }\end{array}$ \\
\hline Moderado (grado 2) & $\begin{array}{c}\text { La presencia de uno o más de los siguientes: } \\
>\quad \text { Recuento elevado de glóbulos blancos } \\
(>18,000 \text { células por milímetro cúbico) }\end{array}$ \\
$>\quad \begin{array}{l}\text { Masa palpable y sensible en el cuadrante } \\
\text { superior derecho. Duración }>72 \mathrm{~h}\end{array}$ \\
\hline
\end{tabular}




\section{Tratamiento y complicaciones de colecistitis calculosa aguda}

Vol. 3, núm. 2., (2019)

Jairo Fernando Bailón Jiménez; Rebeca Isabel Lozano Vera; Piedad Shirley Barragán

Villafuerte; Karen Tatiana Mora Lino

\begin{tabular}{|c|c|c|}
\hline & $D$ & $\begin{array}{l}\text { Inflamación local marcada que incluye } \\
\text { peritonitis biliar, absceso pericolequístico, } \\
\text { absceso hepático, gangreno colecistitis, } \\
\text { colecistitis enfisematosa }\end{array}$ \\
\hline Grave (grado 3) & $\begin{array}{c}\text { La pre } \\
>\end{array}$ & $\begin{array}{l}\text { Dencia de uno o más de los siguientes: } \\
\text { Disfunción cardiovascular (hipotensión } \\
\text { que requiere tratamiento con dopamina a } \\
\geq 5 \mu \mathrm{g} \text { por kilogramo de peso corporal por } \\
\text { minuto o cualquier dosis de dobutamina) } \\
\text { Disfunción neurológica (disminución del } \\
\text { nivel de conciencia) } \\
\text { Disfunción respiratoria (relación entre la } \\
\text { presión parcial de oxígeno arterial y la } \\
\text { fracción de inspiración oxígeno <300) } \\
\text { Disfunción renal (oliguria; nivel de } \\
\text { creatinina,> 2.0 mg / decilitro) } \\
\text { Disfunción hepática (tiempo de } \\
\text { protrombina razón internacional } \\
\text { normalizada, > 1.5) } \\
\text { Disfunción hematológica (recuento de } \\
\text { plaquetas, <100,000 por milímetro } \\
\text { cúbico) }\end{array}$ \\
\hline
\end{tabular}

Fuente: (Takada, Kawarada, \& Nimura, 2007).

\section{Tratamiento}

- Momento de la colecistectomía

La colecistectomía se puede realizar por laparotomía o por laparoscopia, ya sea en el momento del ataque inicial (tratamiento temprano) o de 2 a 3 meses después de que el ataque inicial haya disminuido (tratamiento retrasado). Un factor que complica la evaluación de los resultados del tratamiento temprano, es que "temprano" se ha definido de manera variable como 


\section{Tratamiento y complicaciones de colecistitis calculosa aguda}

Vol. 3, núm. 2., (2019)

Jairo Fernando Bailón Jiménez; Rebeca Isabel Lozano Vera; Piedad Shirley Barragán Villafuerte; Karen Tatiana Mora Lino

entre 24 horas y 7 días después del inicio de los síntomas o el momento del diagnóstico. Si se selecciona un tratamiento retrasado o "conservador", los pacientes son tratados durante la fase aguda con antibióticos y líquidos intravenosos y no se les administra nada por vía oral. Los narcóticos y, en algunos casos, los medicamentos antiinflamatorios no esteroideos se usan para el dolor, y ocasionalmente los pacientes se someten a colecistotostomía percutánea (colocación de un tubo en la vesícula biliar).

La colecistectomía laparoscópica temprana se considera el tratamiento de elección para la mayoría de los pacientes. En ensayos aleatorios y prospectivos comparados en la colecistectomía laparoscópica temprana con un procedimiento retrasado, así como en los metaanálisis de estos ensayos, el tratamiento temprano se ha asociado de manera consistente con una hospitalización general más corta. También favorece la colecistectomía temprana es que aproximadamente del 15 al $20 \%$ de los pacientes que se sometieron a procedimientos retrasados en los ensayos aleatorios tuvieron síntomas persistentes o recurrentes que requirieron intervención antes de su operación planificada. (Lai, 2011)

Los ensayos individuales y los metanálisis tampoco han mostrado diferencias significativas entre los grupos en cuanto a la morbilidad o la mortalidad o en el tiempo operatorio o las tasas de conversión para abrir la colecistectomía. Sin embargo, el tamaño relativamente pequeño de los estudios (el metanálisis más grande involucró solo a 504 pacientes) significa que existe un mayor riesgo de complicaciones poco comunes, en particular, lesión mayor del conducto biliar, una complicación asociada con una morbilidad significativa y, en casos raros, mortalidad, no se puede descartar de manera convincente. 


\section{Tratamiento y complicaciones de colecistitis calculosa aguda}

Vol. 3, núm. 2., (2019)

Jairo Fernando Bailón Jiménez; Rebeca Isabel Lozano Vera; Piedad Shirley Barragán

Villafuerte; Karen Tatiana Mora Lino

Por ejemplo, en la revisión Cochrane, se obtuvieron conclusiones de un total de cuatro lesiones de las vías biliares tomadas entre 438 pacientes $(0,9 \%)$. Las fugas de las vías biliares, una forma de lesión biliar pero considerada por separado, fueron más frecuentes en el grupo que se sometió a procedimientos tempranos $(3.2 \%)$ que en aquellos que se sometieron a procedimientos demorados $(0 \%)$. Los datos de grandes estudios basados en la población sugieren que la lesión biliar es más común cuando la colecistectomía laparoscópica se realiza de forma aguda con vesícula biliar inflamada. (Russell, 2010). También es preocupante que los ensayos pequeños, aleatorios y controlados que compararon la colecistectomía abierta con la colecistectomía laparoscópica no mostraron un mayor riesgo de lesión biliar con la colecistectomía laparoscópica, pero esta tendencia se hizo evidente a través del análisis de grandes registros de pacientes que documentaron complicaciones de procedimiento. Todavía se necesitan estudios con la potencia adecuada para determinar si el momento de la colecistectomía laparoscópica en la colecistitis aguda afecta la tasa de lesiones importantes del conducto biliar.

No se informaron muertes en ninguno de los ensayos aleatorios citados, pero la mortalidad fue superior al $15 \%$ en un estudio reciente de pacientes con colecistitis aguda que estaban en alto riesgo (una puntuación de 12 o más en la Evaluación de Fisiología Aguda y Salud Crónica [APACHE]) (Hatzidakis, 2002). Cuando la colecistectomía laparoscópica se realiza en pacientes con colecistitis aguda moderadamente grave, debe ser realizada por un cirujano altamente experimentado. Si las condiciones quirúrgicas dificultan la identificación anatómica, el procedimiento laparoscópico debe convertirse en una colecistectomía abierta o finalizarse con colecistostomía. 


\section{Tratamiento y complicaciones de colecistitis calculosa aguda}

Vol. 3, núm. 2., (2019)

Jairo Fernando Bailón Jiménez; Rebeca Isabel Lozano Vera; Piedad Shirley Barragán Villafuerte; Karen Tatiana Mora Lino

La tasa de conversión a colecistectomía abierta es más alta cuando se realiza colecistectomía laparoscópica para la colecistitis aguda que para la colelitiasis no complicada, y esto es cierto si la operación se realiza en la fase aguda o después de un retraso. Las tasas de conversión varían desde abajo 5\% a 30\%. Los predictores de la necesidad de conversión incluyen un recuento de glóbulos blancos de más de 18,000 células por milímetro cúbico en el momento de la presentación, una duración de los síntomas de más de un rango de 72 a 96 horas, y una edad mayor de 60 años. (Wong, 2007)

\section{- Terapia antibiótica}

Los fundamentos para el uso de antibióticos y la elección de qué antibiótico usar se basan en los resultados de cultivos biliares de pacientes con colecistitis aguda. Las pautas de la Sociedad de Enfermedades Infecciosas de América recomiendan que se instituya una terapia antimicrobiana si la infección se sospecha sobre la base de hallazgos clínicos y de laboratorio (más de 12,500 glóbulos blancos por milímetro cúbico o una temperatura de más de $38.5^{\circ} \mathrm{C}$ ) y hallazgos radiográficos (por ejemplo, aire en la vesícula biliar o la pared de la vesícula biliar). Dicha terapia debe incluir cobertura contra microorganismos de la familia Enterobacteriana (por ejemplo, una cefalosporina de segunda generación o una combinación de quinolona y metronidazol); no se requiere actividad contra los tercococos. (Mazusk, 2003). Los antibióticos también se recomiendan para el uso de rutina en pacientes de edad avanzada o con diabetes o inmunodeficiencia y para la profilaxis en pacientes sometidos a colecistectomía para reducir las complicaciones sépticas, incluso cuando no se sospecha infección. 


\section{Tratamiento y complicaciones de colecistitis calculosa aguda}

Vol. 3, núm. 2., (2019)

Jairo Fernando Bailón Jiménez; Rebeca Isabel Lozano Vera; Piedad Shirley Barragán

Villafuerte; Karen Tatiana Mora Lino

\section{- Colecistostomía percutánea}

La colecistostomía percutánea que se realiza bajo anestesia local con guía radiológica a menudo se usa cuando el paciente presenta sepsis (colecistitis aguda grave, según las directrices de Tokio) y en los casos en que el tratamiento conservador solo falla, especialmente en pacientes que son malos candidatos para la cirugía. La colecistostomía percutánea tiene una alta tasa de éxito técnico y una baja tasa de complicaciones y generalmente resulta en la resolución de la colecistitis aguda (McGahan, 2014). Sin embargo, en un ensayo aleatorio en pacientes de alto riesgo, la colecistostomía percutánea de rutina no fue superior a las medidas conservadoras seguidas de colecistostomía percutánea cuando fue necesario. El drenaje puede ser seguido por colecistectomía retrasada o extracción de cálculos percutáneos en pacientes que son candidatos quirúrgicos pobres. La colecistostomía operatoria se usa cuando la colecistectomía no puede completarse debido a condiciones operativas difíciles.

\section{Conclusiones.}

Las directrices de Tokio proporcionan recomendaciones para el tratamiento en función de la gravedad de la colecistitis aguda. Para la colecistitis aguda leve, se recomienda la colecistectomía laparoscópica temprana. Para la colecistitis aguda moderada, las pautas indican que puede seleccionarse la colecistectomía temprana o tardía, pero que la colecistectomía laparoscópica temprana debe ser realizada solo por un cirujano altamente experimentado y debe terminar rápidamente por conversión a colecistostomía abierta si las condiciones operativas hacen anatómica. 


\section{Tratamiento y complicaciones de colecistitis calculosa aguda}

Vol. 3, núm. 2., (2019)

Jairo Fernando Bailón Jiménez; Rebeca Isabel Lozano Vera; Piedad Shirley Barragán Villafuerte; Karen Tatiana Mora Lino

La identificación difícil en una minoría de pacientes con colecistitis aguda grave, se recomienda el tratamiento conservador inicial con antibióticos, preferiblemente en un entorno de alta agudeza, con el uso de colecistostomía percutánea según sea necesario; la cirugía está reservada para pacientes en quienes este tratamiento falla. Las pautas de la Sociedad de Enfermedades Infecciosas de América para el uso de antibióticos en la colecistitis aguda se analizan durante el desarrollo del proceso investigativo y su preescripcion de basa de acuerdo a los resultados de los cultivos biliares de los pacientes con colecistitis aguda.

\section{Bibliografía.}

Blaivas, M., \& Adhikari, S. (2007). Diagnostic utility of cholescin-tigraphy in emergency department pa-tients with suspected acute cholecystitis: comparison with bedside RUQ ultraso-nography. J Emerg Med, 47-52.

Bolívar, J. (2015). Investigación Documental. México. Pax.

Carter, Cox, \& Polk. (2013). Opera-tive therapy for cholecystitis and choleli-thiasis: trends over three decades. Am Surg.

Castro, J. (2016). Técnicas Documentales. México. Limusa.

Claesson, Holmlund, \& Mätzsch. (2014). Microflora biliar en la colecistitis aguda y las implicaciones clínicas. Acta Chir Scand, (págs. 229-37.). Medellin.

Davila, A. (2015). Diccionario de Términos Científicos. Caracas: Editorial Oasis.

Gurusamy, \& Samraj. (2006). Early versus delayed laparoscopic cholecystectomy for acute cholecystitis. Cochrane Database Syst Rev.

Hatzidakis. (2002). Acute cholecystitis in high-risk patients: percutaneous chole-cystostomy vs conservative treatment. Eur Radiol, 60-84.

Hicks, R., Kelly, M., \& V, K. (2011). Association between false negative hepatobiliary scans and initial gallbladder visualization after. Los Angeles.

Juvonen, T., Kiviniemi, H., Niemelä, O., \& Kairaluoma, M. (2007). Diagnostic accuracy of ultrasonography and $\mathrm{C}$ reactive protein Biliary microflora in acute cholecystitis and the clinical implications. Acta Chir Scand (págs. 229-37.). Santiago: Acta Chir Scand.

Juweid, M., \& Mozley, P. (2003). Cholescintigraphy in the diagnosis of acute cholecystitis: morphine augmentation is superior to de-layed imaging. . Washington: J Nucl Med.

Lai, P. (2011). Ensayo aleatorio de colecistectomía laparoscópica temprana versus tardía para colecistitis aguda. Buenos Aires: Br J Surg.

Mazusk, J. (2003). Pautas para la selección de agentes antiinfecciosos para infecciones intraabdominales complicadas. Clin Infect Dis, 56-89. 


\section{Tratamiento y complicaciones de colecistitis calculosa aguda}

Vol. 3, núm. 2., (2019)

Jairo Fernando Bailón Jiménez; Rebeca Isabel Lozano Vera; Piedad Shirley Barragán

Villafuerte; Karen Tatiana Mora Lino

McGahan, C. (2014). Percutaneous cholecystostomy: an alternative to surgical cholecystostomy for acute chole-cystitis? Radiology. 45-89.

Morrow, D., Thompson, J., \& Wilson, S. (2008). Colecistitis aguda en ancianos: una emergencia quirúrgica. Bogota: Arch Surg.

Ralls, W. (2013). Ecografía en tiempo real en sospecha de colecistitis aguda: evaluación prospectiva de signos primarios y secundarios. Caracas: Radiología.

Roslyn, DenBesten, Thompson, \& Silverman. (2010). Roles de la bilis litogénica y la oclusión de los conductos quísticos en el patógeno-esis de la colecistitis aguda. Am J Surg, 126-30.

Russell, C. (2010). Lesiones del conducto biliar, 1989-1993: una experiencia en todo el estado: Registro de colecistectomía laparoscópica de Connecticut. (págs. 382-8). Arch Surg.

Takada, Kawarada, \& Nimura. (2007). Background:Tokyo guidelines for the management of acute cholangitis and cholecystitis. J Hepatobiliary Pancreat Surg. Los Angeles.

Wong, J. (2007). Early decision for conversion of laparo-scopic to open cholecystectomy for treatment of acute cholecystitis. Am J Surg, 7-13.

Yeatman, J. (2009). Emphysematous chole-cystitis: an insidious variant of acute cho-lecystitis. Los Angeles: Am J Emerg Med.

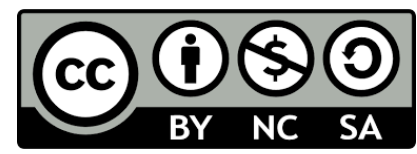

RECONOCIMIENTO-NOCOMERCIAL-COMPARTIRIGUAL

CC BY-NC-SA

ESTA LICENCIA PERMITE A OTROS ENTREMEZCLAR, AJUSTAR Y CONSTRUIR A PARTIR DE SU OBRA CON FINES NO COMERCIALES, SIEMPRE Y CUANDO LE RECONOZCAN LA AUTORÍA Y SUS NUEVAS CREACIONES ESTÉN BAJO UNA LICENCIA CON LOS MISMOS TÉRMINOS. 